\title{
Is Target Oriented Surgery Sufficient with Borderline Ovarian Tumors? - Role of Accompanying Pathologies
}

\author{
Tayfun Gungor ${ }^{1}$, Nilufer Cetinkaya ${ }^{1 *}$,Hakan Yalcin ${ }^{1}$, Bulent Ozdal $^{1}$, Emre Ozgu $^{1}$, \\ Eralp Baser ${ }^{1}$, Nafiye Yilmaz ${ }^{1}$, Mete Caglar $^{2}$, Sema Zergeroglu ${ }^{3}$, Salim Erkaya ${ }^{1}$
}

\begin{abstract}
Background: There are limited data in the literature related to concomitant genital or extra-genital organ pathologies in patients with borderline ovarian tumors (BOTs). The aim of this study was to evaluate our experience with 183 patients to draw attention to the accompanying organ pathologies with BOTs. Materials and Methods: One hundred eighty-three patients with BOTs, diagnosed and/or treated in our center between January of 2000 and March of 2013 were evaluated retrospectively. Data related to age, tumor histology, lesion side, disease stage, accompanying incidental ipsilateral and/or contralateral ovarian pathologies, treatment approaches, and follow-up periods were investigated. Incidental gynecologic and non-gynecologic concomitant organ pathologies were also recorded. Results: The mean age at diagnosis was 40.6 years (range: 17-78). Ninetyfive patients $(\mathbf{5 1 \%})$ were $\leq 40$ years. A hundred and forty-seven patients $(\mathbf{8 0 \%})$ were at stage IA of the disease. The most common type of BOT was serous in histology. Non-invasive tumor implants were diagnosed in $4 \%$ and uterine involvement was found $2 \%$ among patients who underwent hysterectomies. There were 12 patients with positive peritoneal washings. Only 17 and 84 patients respectively had concomitant ipsilateral and concomitant contralateral incidental ovarian pathologies. The most common type of uterine, appendicular and omental pathologies were chronic cervicitis, lymphoid hyperplasia and chronic inflammatory reaction. Conclusions: According to our findings most of accompanying pathologies for BOT are benign in nature. Nevertheless, there were additional malignant diseases necessitating further therapy. We emphasize the importance of the evaluation of all abdominal organs during surgery.
\end{abstract}

Keywords: Borderline ovarian tumors - ovarian carcinoma - accompanying pathologies - staging - fertility sparing

Asian Pac J Cancer Prev, 15 (16), 6749-6754

\section{Introduction}

Borderline ovarian tumors (BOTs) account for about $10-20 \%$ of epithelial ovarian malignancies. These tumors have epithelial proliferations with mild atypia and without stromal invasion except for micro-invasive variants that have one or more foci of stromal invasion $\leq 3 \mathrm{~mm}$ (Seidman et al., 2004).

BOTs diagnosed in young women tend to have better prognosis with 90\%-100\% survival rates (Ayhan et al., 2005; Trope et al., 2012). Patients might present with pelvic pain, abdominal fullness, increased urination or cystic mass diagnosed with imaging. Tumor markers increase in roughly $25-60 \%$ of patients (Poncelet et al., 2010). The risk of malignancy index (RMI) calculated by the serum levels of CA-125, ultrasonographic score, and menopausal status during patient evaluation is able to discriminate between benign and BOTs (Moolthiya and Yuenyao, 2009; Arun-Muthuvel and Jaya, 2014). Modified cutoff values of CA-125 and RMI score were reported to result in a better prediction of malignancy, at the cost of reduced sensitivity in patients with ovarian masses (Winarto et al., 2014). Furthermore, tissue levels of CA-125 and HE4 proteins were reported to be higher in ovarian malignancies than in benign lesions (Devan et al., 2013) and elevated CA-125 levels in blood is known to predict tumor burden ( $\mathrm{Li}$ et al., 2012).

BOTs are surgically staged according to the International Federation of Gynecology and Obstetrics (FIGO) 2009 staging system. Most patients with BOTs are diagnosed as stage I of the disease. However, BOTs might have invasive or non-invasive implants spreading onto the surrounding pelvic or abdominal organs and if present increase the stage of disease (Menczer et al., 2012). Surgical treatment is the same as for malignant ovarian tumors but there is no difference in the recurrence

${ }^{1}$ Department of Gynecologic Oncology, ${ }^{3}$ Department of Pathology, Zekai Tahir Burak Women's Health Education and Research Hospital, Ankara, ${ }^{2}$ Department of Obstetrics and Gynecologic, Faculty of Medicine, Düzce University, Düzce, Turkey *For correspondence: cetinkayanilufer@gmail.com 
or survival rate whether lymphadenectomy is performed or not. Therefore, lymphadenectomy can be postponed (Kanat-Pektas et al., 2011b). Laparoscopic surgery is a suitable choice in early stages of the disease (Tinelli et al., 2009). Protection of fertility by preserving the uterus and contralateral ovary is preferable for young patients (Karimi Zarchi et al., 2011). Unilateral oophorectomy or cystectomy are safe options for the stage I disease if careful follow-up of the patient is possible (Tinelli et al., 2006; Yinon et al., 2007; Park et al., 2009). However, the recurrence rate is $12 \%-58 \%$ after cystectomy (Trope et al., 2012). Hysterectomies with bilateral salpingooophorectomy are performed in women at more advanced ages (Menczer et al., 2012). The accuracy of intraoperative frozen section for the diagnosis of ovarian masses is high (Suprasert et al., 2008). However, the sensitivity of the frozen section for BOTs is about $45 \%-64 \%$ (Wu et al., 2009; Poncelet et al., 2010), which means that some patients are diagnosed in permanent pathology. Because it is known that lymph node involvement might be $20 \%$ in stage I serous BOTs (Trope et al., 2012), restaging surgery is questionable.

Spontaneous pregnancies usually occur after conservative surgery and pregnancy outcomes are known to be promising (Nam, 2010). Young age at diagnosis, non-serous histology and unilateral cystectomy are known to be associated with favorable reproductive outcomes in women on whom conservative surgery was performed (Kanat-Pektas et al., 2011a).

BOT's are relatively resistant to chemotherapy and there is little effect on long-term survival in advanced stage disease due to their low-malignant potential (Trope et al., 2012). Micro-invasive or micro-papillary histology, conservative procedures like cystectomy or unilateral salpingo-oophorectomy, cyst rupture, advanced stage disease and the presence of tumor implants are prognostic factors affecting recurrence (Wu et al., 2009).

There are numerous papers in the literature related with the diagnosis, treatment options, postoperative surveillance, recurrence and prognostic factors of BOTs. However, there are limited data concerning the accompanying organ pathologies with BOTs in these patients (Kanat-Pektas et al., 2010). The aim of this study was to evaluate our experience with 183 patients having been diagnosed with BOTs and to draw attention to concomitant ipsilateral and contralateral ovarian, uterine, omental, appendiceal, lymph nodal, peritoneal or nongynecologic organ abnormalities.

\section{Materials and Methods}

The present study was approved by the Institutional Review Board of Zekai Tahir Burak Women' Health Education and Research Hospital where the study was conducted.

183 patients with BOTs, diagnosed and/or treated in our center between January of 2000 and March of 2013 were included in the study. Clinical and pathological data were reviewed retrospectively. Permanent pathology reports of patients were analyzed and concomitant pathologic lesions with BOTs were characterized.
From the hospital records of the 183 patients, data related with age, type of surgery, disease stage, primary BOT lesion side, tumor diameter, tumor histology, accompanying ipsilateral and contralateral ovarian lesions diagnosed incidentally, results of peritoneal washings, presence of and characteristics of tumor implants, dissected lymph node counts were reviewed. Furthermore, coexistent pathologies of the uterus, lymph nodes, omentum, appendix and peritoneum were evaluated. Also the presence of non-gynecological pathologies were noted. The mean postoperative follow-up periods, data related with the disease recurrence and conditions necessitating recurrent operations were commentated as secondary inferences.

The patients were staged surgically according to FIGO 2009 guidelines for ovarian carcinoma. Comprehensive surgical staging with peritoneal sampling, total abdominal hysterectomy, bilateral salpingo-oophorectomy, pelvic and para-aortic lymphadenectomy, appendectomy and omentectomy were performed in patients who were postmenopausal, completed their fertility or had additional disease that require extensive surgery. Fertility sparing surgery was performed in the form of unilateral salpingooopherectomy (USO), cystectomy, USO with contralateral ovarian biopsy, USO with contralateral cystectomy, cystectomy with contralateral ovarian biopsy and bilateral ovarian biopsy in patients who were premenopausal or wish to preserve their fertility.

Statistical analysis

The statistical analysis was performed with SPSS for Mac version 20 (SPSS for Mac Inc., Chicago, IL, US). We computed descriptive statistics (mean and range). An independent samples t test was performed to compare the difference between the tumors' histologic types.

\section{Results}

A total of 183 patients were diagnosed. The mean age at diagnosis was 40.6 (range: $17-78$ ). Ninety-five patients $(51 \%)$ were $\leq 40$ years. A total of 91 hysterectomies, 176 peritoneal fluid washings, 153 appendectomies, 163 omentectomies and 159 pelvic \& para-aortic lymph node dissections were performed.

Comprehensive surgical staging was performed on 91 patients (49\%). There were 14 patients in the comprehensive staging surgery group that were $\leq 40$

Table 1. Data for Ovarian Tumors

\begin{tabular}{llrr}
\hline Location & & & Total \\
\hline Right Ovary & Serous Bot & 67 & 113 \\
& Serous Micro-Invasive Bot & 5 & \\
& Serous Micro-Papillary Bot & 2 & \\
& Mucinous Bot & 33 & \\
& Endometrioid Bot & 5 & \\
& Clear Cell Bot & 1 & \\
Left Ovary & Serous Bot & 59 & 93 \\
& Serous Micro-Invasive Bot & 3 & \\
& Serous Micro-Papillary Bot & 1 & \\
& Mucinousbot & 27 & \\
& Mucinous Micro-Invasive Bot & 1 & \\
& Brenner Bot & 2 & \\
\hline Total & & & 206 \\
\hline
\end{tabular}


years old and had hysterectomies due to concomitant uterine pathologies. The reasons for the hysterectomies were secondary dysmenorrhea and abnormal uterine bleeding due to giant or multiple myoma, adenomyosis, endometrial polyps or simple endometrial hyperplasia without atypia. Fertility-sparing surgery with unilateral salpingo-oophorectomy or cystectomy, with or without contralateral ovarian biopsy, was performed in $48 \%$ of the patients (n: 89). The mean age of the patients in the fertility-sparing group was significantly lower than patients in comprehensive surgical staging group -30 years (range: 17-45) and 50 years (range: $31-78$ ) respectively. Lymph node dissection was done in $76 \%$ of these patients (n: 68). Furthermore, unilateral salpingo-oopherectomy (USO), cystectomy, USO with contralateral ovarian biopsy, USO with contralateral cystectomy, cystectomy with contralateral ovarian biopsy and bilateral ovarian biopsy were performed in 38, 18, 27, 3, 2 and 1 patient respectively.

The patients that had complete surgical staging or fertility sparing surgery with lymph node dissection were operated on using a midline abdominal incision. Patients that had fertility sparing surgery without lymph node dissection (n: 21) were operated on using a pfannenstiel incision. These patients all had negative preoperative tumor markers.

The disease stages at surgery were stage IA $(80 \%, \mathrm{n}$ : 147), stage IB (9\%, n: 17), stage IC (1\%, n: 3), stage IIC $(2 \%, \mathrm{n}: 4)$ and stage IIIC (6\%, n: 12$)$ respectively. The most common type of BOT was serous in histology with $18 \%$ bilateralism followed by mucinous, endometrioid, Brenner and clear cell. Another patient had synchronous right-sided serous micro-invasive and left sided mucinous BOT on separate ovaries. Ninety patients $(49 \%)$ had unilateral right and 70 patients $(38 \%)$ had unilateral left sided BOT. Twenty-three patients' BOT lesions were bilateral $(12 \%)$. Serous BOT was the leading $(91 \%)$ histologic type in bilateral tumors. There were 113 BOTs on the right ovary and 93 BOTs on the left ovary. Number of patients and ovarian lesions in respect to

tumor localizations and histological characteristics are summarized in Tables 1 and 2.

The mean tumor diameter was $9.4 \mathrm{~cm}$ (range: 1-27), $14.8 \mathrm{~cm}$ (range: $3-38$ ), $7.6 \mathrm{~cm}$ (range: $0.8-13), 10.5 \mathrm{~cm}$ (range: 9-12) in serous, mucinous, endometrioid and Brenner type BOT respectively. The tumor diameter of the clear cell BOT was $13 \mathrm{~cm}$. Mucinous BOTs had significantly larger tumor diameters than the serous BOTs $(\mathrm{P}<0.0001)$.

There were 12 patients (6\%) with positive peritoneal washings. The cytology reports of 9 patients were correlated with serous BOT and 3 patients' reports were correlated with malignancy ( 2 with bilateral serous and one with bilateral mucinous BOTs). Hemorrhagic contaminations, mesothelial proliferations or subacute inflammatory reactions were other subtle changes.

Non-invasive tumor implants were diagnosed in 9 patients (4\%); 5 with serous, 2 with serous micro-papillary and 2 with mucinous BOTs. Implants were mostly located on the Douglas pouch, uterine or tubal serosa, bladder serosa, sigmoid mesocolon, omentum, small intestine mesentery and the area surrounding the ureter.

The mean number of dissected lymph nodes was 56.98 (range: $12-173$ ). Of the 159 patients' lymph node dissections, only $10(6 \%)$ had lymph node abnormalities other than lymphoid hyperplasia. Four of these patients had benign glands, lined by tubal-type epithelium (also called endosalpingiosis); 3 had benign inclusional glandular implants; 2 showed the presence of serous BOTs; and one, who had synchronous serous adenocarcinoma on the contralateral ovary, had tumor-positive lymph nodes.

Of the 90 patients who had right unilateral BOTs and the 70 patients who had left unilateral BOT, only 12 and 5 patients respectively had concomitant ipsilateral incidental ovarian pathology. Moreover, of the 90 patients who had right unilateral BOTs only 53 patients had concomitant contralateral incidental ovarian pathology and of the 70 patients who had left unilateral BOTs only 31 patients had it. Table 3 and 4 presents the accompanying ipsilateral and contralateral incidental ovarian pathologies with BOTs.

Table 2. Pathological Diagnoses of Ovarian Tumors

\begin{tabular}{|c|c|c|c|}
\hline \multicolumn{4}{|c|}{ Incidental Ipsilateral Ovarian Lesions } \\
\hline Primary Right Ovarian Tumors & & Incidental Right Ovarian Lesions & \\
\hline \multirow[t]{5}{*}{ Serous Bot } & 6 & Endosalpingiosis & 2 \\
\hline & & Cystadenofibroma & 1 \\
\hline & & Endometrioma & 1 \\
\hline & & Serous Cystadenoma, Endometrioma & 1 \\
\hline & & Synchronous Intra-Ovarian Serous Bot & 1 \\
\hline \multirow[t]{3}{*}{ Mucinous Bot } & 3 & Endometrioma & 1 \\
\hline & & Mucinous Cystadenoma, Mucinous Adenocarcinoma & 1 \\
\hline & & Endometrioma, Mature Cystic Teratoma & 1 \\
\hline \multirow[t]{2}{*}{ Endometrioid Bot } & 2 & Endometrioid Carcinoma & 1 \\
\hline & & Endometrioid Carcinoma, Endometrioid Adenofibroma & 1 \\
\hline Clear Cell Bot & 1 & Clear Cell Carcinoma, Endometrioma, Adenofibroma & 1 \\
\hline \multicolumn{2}{|l|}{ Total } & & 12 \\
\hline \multirow{4}{*}{$\begin{array}{l}\text { Primary Left Ovarian Tumors } \\
\text { Serous Bot } \\
\text { Mucinous Bot }\end{array}$} & & Incidental Left Ovarian Lesions & \\
\hline & 2 & Endometrioma & 2 \\
\hline & 2 & Mucinous Cystadenocarcinoma & 1 \\
\hline & & Benign Brenner Tumor & 1 \\
\hline Brenner Bot & 1 & Benign Brenner Tumor & 1 \\
\hline \multicolumn{2}{|l|}{ Total } & & 5 \\
\hline
\end{tabular}


There were no concomitant ovarian pathologies for the 2 Brenner BOT on left or right ovary. Follicular cyst, corpus luteum and inclusional cyst were the other benign findings diagnosed in paraffin blocs of the ovarian specimens.

Hysterectomies were performed on 91 patients. Five patients had normal appearing uterine specimens with proliferative or secretory endometrial lining. The most common types of uterine pathologies were chronic cervicitis $(63.7 \%, \mathrm{n}: 58)$ followed by myoma uteri $(37.3 \%, \mathrm{n}: 34)$ and adenomyosis (24.1\%, n: 22$)$. Thirteen patients had concomitant endometrial polyps, 4 patients had low-grade and 2 had high-grade squamous intraepithelial lesions. Three patients-one with bilateral serous BOTs, one with bilateral micro-papillary serous BOTs and one with bilateral mucinous BOTs-had tubal, para-tubal and/or uterine serosal non-invasive implants. The concomitant uterine pathologies of another 4 patients with serous BOTs were: degenerated placental tissues (BOT was diagnosed during cesarean section), bilateral adnexal endosalpingiosis, para-tubal serous carcinoma at the same site as the BOT and tubal malign mixed mullerian tumor (MMMT) involvement of clear-endometrioid-leiomyosarcoma histology due to accompanying contralateral ovarian MMMT. A chronic granulomatous lesion in the endometrium and on the cervix with bilateral tubal salpingitis folicularis due to
Tuberculosis was another entity that accompanied the unilateral Brenner BOT-Benign Brenner combination. Salpingitis, serosal endometriotic implants, endometritis and simple hyperplasia without atypia were the other less encountered uterine lesions.

The appendectomy specimens of 88 patients had normal histology $(57.5 \%)$. The most common types of appendix pathology were lymphoid hyperplasia (18.9\%, n: 29, one with local peritonitis) followed by peri-appendicitis (13.7\%, n: 21) and luminal obliteration $(7.1 \%, \mathrm{n}: 11)$. Only one patient had acute inflammation of the appendix. A case with bilateral mucinous BOTs had incidental appendiceal mucinous cyst-adenoma and large spread peritoneal, omental mucinous tumor implantation, which resulted in Disseminated Peritoneal Adenomucinosis. Another patient who had unilateral serous BOT also had a $1.5 \mathrm{~cm}$ insular type appendiceal carcinoid tumor. Endosalpingiosis, the presence of a reactive lymph node and enterobiasis infection were the other less encountered types of appendiceal lesions.

The omentectomy specimens of 129 patients (79.1\%) had normal histology as a mature adipose tissue. Chronic inflammatory reaction $(6.1 \%, \mathrm{n}: 10)$, a reactive lymph node $(4.9 \%, \mathrm{n}: 8)$, subacute peritonitis $(2.4 \%, \mathrm{n}: 4)$, mesothelial proliferation $(1.8 \%, \mathrm{n}: 3)$ and inclusional gland implantation $(1.2 \%, \mathrm{n}: 2)$ were the most commonly

Table 3. Tumor Types Encountered

Incidental Contralateral Ovarian Lesions Primary Right Ovarian Tumors

\begin{tabular}{lccc}
\hline Serous Bot & 34 & Serous Bot & 18 \\
& & Serous Cystadenoma & 7 \\
& & Endometrioma & 5 \\
& & Endometrioid Carcinoma & 1 \\
& & Ovarian Malign Mixed Mullerian Tumor & 1 \\
& & Adenofibroma & 1 \\
& & Fibromatous Nodule & 1 \\
Serous Micro-Invasive Bot & 3 & Serous Bot & 1 \\
& & Serous Micro-Invasive Bot & 1 \\
Serous Micro-Papillary Bot & & Mucinous Bot & 1 \\
Mucinous Bot & 1 & Serous Micro-Papillary Bot & 1 \\
& 13 & Endometrioma & 4 \\
& & Mucinous Cystadenoma & 4 \\
& & Serous Cystadenoma & 3 \\
Endometrioid Bot & Mucinous Bot & 1 \\
& & Fibroma & 1 \\
Total & 2 & Endometrioma, Endometrioid Carcinoma & 1 \\
\hline
\end{tabular}

Total

Primary Left Ovarian Tumors

Serous Bot

Serous Micro-Invasive Bot

Serous Micro-Papillary Bot

Mucinous Bot
Incidental Left Ovarian Lesions

Incidental Right Ovarian Lesions

Serous Bot

18

Serous Cystadenoma

3

Serous Carcinoma

2

Serous Micro-Invasive Bot 1

Ovarian Micro Abscess 1

Endometrioma 1

Serous Micro-Invasive Bot 1

Serous Micro-Papillary Bot $\quad 1$

Mucinous Bot 1

Mature Cystic Teratoma, Mucinous Cyst 1

Serous Micro-Invasive Bot 
encountered lesions. Only 3 patients $(1.8 \%)$ had noninvasive omental BOT implants. Metastasis from gastric carcinoma $(0.6 \%)$, mucinous tumor infiltration $(0.6 \%)$ due to disseminated peritoneal adenomucinosis, endosalpingiosis and chronic granulomatous process accompanying bilateral mucinous BOT were the other less encountered omental lesions.

In addition to the aforementioned conditions, there were three patients who had bilateral serous BOTs and extra-ovarian incidental pathologies. The first patient had pathologically confirmed renal methanephric adenoma that was diagnosed during follow-up period shortly after the staging surgery. The second patient had synchronous lymhoepithelioma type gastric carcinoma, which was diagnosed per-operatively during staging surgery, and she also had a pelvic cyst diagnosed as hydatic cyst in permanent pathology. The third patient had diffuse noninvasive peritoneal serous BOT implants with retroperitoneal desmoplasia and sarcomatous mural nodules with increased mitosis and necrosis accompanying adnexal tumor mass. Furthermore, another patient with unilateral mucinous BOT had a suspicious peritoneal biopsy, which revealed accessory spleen and Meckel diverticulum was another incidental condition associated with unilateral serous BOT.

The mean postoperative follow-up period was 20.4 months (range: 6-78 months). During the follow-up period, 15 patients that had accompanying malignancy, peritoneal implants and/or lymph node positivity had post-operative chemotherapy. Disease recurrence was seen in 5 of the 183 patients, indicating an overall recurrence rate of $2.7 \%$. All of the recurrences except one were diagnosed after fertility sparing surgery. Recurrent tumors had serous histology. Contralateral ovarian recurrence was seen in two patients who had had USO previously. One patient who had previously had a cystectomy experienced ipsilateral ovarian recurrence. Another patient with a history of cystectomy and chemotherapy due to serous BOT with implants had recurrent benign ovarian cyst and pelvic non-invasive BOT implants. There was only one patient in the comprehensive surgery group whose disease recurred after debulking surgery. Recurrence was in the form of bilateral cystic masses simulating slowly enlarging lymphocysts with normal tumor markers.

During the follow-up period, only 9 patients had secondary operations: five due to disease recurrence, one with recurrent serous ovarian malignancy with a history of serous BOT and concomitant serous ovarian malignancy, one with benign ovarian cyst on the contralateral ovary with mucinous BOT history, one with bilateral benign ovarian cyst with a history of serous BOT and another due to the formation of a $10 \mathrm{~cm}$ lymphocyst with a history of mucinous BOT. Only two patients from the comprehensive surgery group had secondary surgery.

\section{Discussion}

In our study, we evaluated a large data pool of 183 patients diagnosed as borderline epithelial ovarian tumor. There were 95 patients $(51 \%)$ aged $\leq 40$. Our results indicate that BOTs tend to be diagnosed at a younger age, which is consistent with the literature (Gotlieb et al., 2005; Romagnolo et al., 2006). Serous BOTs were more numerous than mucinous ones and endometrioid, Brenner and clear cell BOTs were diagnosed relatively infrequently. Bilateral tumors more commonly had serous histology, and mucinous tumors were larger than serous ones. These results were compatible with the literature (McCluggage, 2010; Messalli et al., 2013).

There might be accompanying genital and extragenital organ pathologies with BOTs (Gungor et al., 2011). However the data in the literature related with this topic is quite limited and the issue is addressed only in several case reports. According to our study, most of the accompanying pathologies with BOTs were ovarian in origin and benign in nature. Serous cystadenoma, endometrioma, mucinous cyst, adenofibroma and mature cystic teratoma were the most commonly encountered ovarian lesions concomitant to BOTs. Nevertheless, we demonstrated that there could be additional malignant tumor foci within the BOTs. Such as, serous, mucinous, endometrioid or clear cell carcinoma. Based on our findings, the minimum tumor diameters of the incidental ipsilateral and contralateral ovarian malignancies accompanying to BOTs were reported to be $8 \mathrm{~mm}$ and $1.2 \mathrm{~cm}$ respectively. Furthermore we also demonstrated the presence of malignant tumors that had different cellular in origins. Such as, gastric carcinoma, appendiceal carcinoid and mixed Mullerian tumor. The complex association of BOTs with genital or extra-genital organ pathologies leads to figure out the importance of the tissue sampling during operation and the macroscopic evaluation of the sampled tissue after surgery.

Ovarian serous carcinoma, endometrioid carcinoma, ovarian malign mixed Mullerian tumor, mucinous carcinoma and clear cell carcinoma were associated with BOTs as ipsilateral or contralateral incidental coexistent ovarian lesions. The presence of invasive disease required treatment manipulation against malignancy in these patients. Nearly $66 \%$ of patients that required postoperative chemotherapy had the incidental concomitant ovarian, para-tubal or gastric malignancy and the other $13 \%$ had the incidental contralateral ovarian micro-invasive BOTs.

We observed that peritoneal fluid abnormality, lymph node positivity and uterine involvement with BOTs were $6 \%, 6 \%$ and $2 \%$ respectively. Uterine pathologies were generally benign in nature except for patients with cervical squamous intraepithelial lesions, para-tubal serous carcinoma and tubal malign mixed Mullerian tumors. Furthermore, the omentum and appendix were the less encountered organs with pathologies concomitant to BOTs and the presence of the BOT implants on the omentum and the carcinoid tumor of the appendix were their most remarkable pathologies.

To our knowledge this study is the only one in the literature that evaluates a large data pool of patients to point out the concomitant organ pathologies with BOTs. In spied of its retrospective nature based on the results of our study, we emphasize the importance of the visualization and palpation of all abdominal organs during staging surgery for BOTs. The surgeon's ability to recognize suspicious areas and request a biopsy during surgery enables incidental pathologies to be diagnosed, which 
might have a positive effect on the patients' survival. Also it must be remembered that fertility-sparing surgery in patients with BOTs and the presence of incidental malignancies impact on the disease recurrence.

Patient follow-up after detection of accompanying pathologic conditions must be individualized. Such as, the presence of a concomitant squamous cell intraepithelial lesion must preclude Pap test screening for early detection of invasive squamous cell pathologies originating from the vaginal cuff or the vagina itself. Close monitoring after primary therapy is essential for the diagnosis of disease recurrence. Benign pathologies might be seen on close follow-up. However, malignant processes or recurrent BOTs are need to be diagnosed, especially after invasive serous tumors or serous BOTs with invasive or non-invasive tumor implants such as those that occurred in our study. However, further prospective studies with larger numbers and detailed documentations of the accompanying pathologies with BOTs are needed to more clearly delineate this issue.

\section{References}

Arun-Muthuvel V, Jaya V (2014). Pre-operative evaluation of ovarian tumors by risk of malignancy index, CA125 and ultrasound. Asian Pac J Cancer Prev, 15, 2929-32.

Ayhan A, Guvendag Guven ES, Guven S, et al (2005). Recurrence and prognostic factors in borderline ovarian tumors. Gynecol Oncol, 98, 439-45.

Devan SM, Pailoor J, Sthaneshwar P, et al (2013). Pattern of tissue expression of CA-125 and HE4 in primary epithelial ovarian tumours and correlation with serum CA-125 levels. Asian Pac J Cancer Prev, 14, 4545-8.

Gotlieb WH, Chetrit A, Menczer J, et al (2005). Demographic and genetic characteristics of patients with borderline ovarian tumors as compared to early stage invasive ovarian cancer. Gynecol Oncol, 97, 780-3.

Gungor T, Altinkaya SO, Sirvan L, et al (2011). Coexistence of borderline ovarian epithelial tumor, primary pelvic hydatid cyst, and lymphoepithelioma-like gastric carcinoma. Taiwan J Obstet Gynecol, 50, 201-4.

Kanat-Pektas M, Ozat M, Gungor T, et al (2011a). Fertility outcome after conservative surgery for borderline ovarian tumors: a single center experience. Arch Gynecol Obstet, 284, 1253-8.

Kanat-Pektas M, Ozat M, Gungor T, et al (2011b). Complete lymph node dissection: is it essential for the treatment of borderline epithelial ovarian tumors? Arch Gynecol Obstet, 283, 879-84.

Kanat-Pektas M, Yenicesu O, Gungor T, et al (2010). Predictive power of sexual hormones and tumor markers in endometrial cancer. Arch Gynecol Obstet, 281, 709-15.

Karimi Zarchi M, Mousavi A, Gilani MM, et al (2011). Fertility sparing treatments in young patients with gynecological cancers: Iranian experience and literature review. Asian Pac J Cancer Prev, 12, 1887-92.

$\mathrm{Li} \mathrm{L,} \mathrm{Xu} \mathrm{Y,} \mathrm{Yu} \mathrm{CX} \mathrm{(2012).} \mathrm{Proteomic} \mathrm{analysis} \mathrm{of} \mathrm{serum} \mathrm{of} \mathrm{women}$ with elevated Ca-125 to differentiate malignant from benign ovarian tumors. Asian Pac J Cancer Prev, 13, 3265-70.

McCluggage WG (2010). The pathology of and controversial aspects of ovarian borderline tumours. Curr Opin Oncol, 22, 462-72.

Menczer J, Chetrit A, Sadetzki S (2012). The effect of hysterectomy on survival of patients with borderline ovarian tumors. Gynecol Oncol, 125, 372-5.
Messalli EM, Grauso F, Balbi G, et al (2013). Borderline ovarian tumors: features and controversial aspects. Eur J Obstet Gynecol Reprod Biol, 167, 86-9.

Moolthiya W, Yuenyao P (2009). The risk of malignancy index (RMI) in diagnosis of ovarian malignancy. Asian Pac J Cancer Prev, 10, 865-8.

Nam JH (2010). Borderline ovarian tumors and fertility. Curr Opin Obstet Gynecol, 22, 227-34.

Park JY, Kim DY, Kim JH, et al (2009). Surgical management of borderline ovarian tumors: The role of fertility-sparing surgery. Gynecol Oncol, 113, 75-82.

Poncelet C, Fauvet R, Yazbeck C, et al (2010). Impact of serum tumor marker determination on the management of women with borderline ovarian tumors: multivariate analysis of a French multicentre study. Eur J Surg Oncol, 36, 1066-72.

Romagnolo C, Gadducci A, Sartori E, et al (2006). Management of borderline ovarian tumors: results of an Italian multicenter study. Gynecol Oncol, 101, 255-60.

Seidman JD, Soslow RA, Vang R, et al (2004). Borderline ovarian tumors: diverse contemporary viewpoints on terminology and diagnostic criteria with illustrative images. Hum Pathol, 35, 918-33.

Suprasert P, Khunamornpong S, Phusong A, et al (2008). Accuracy of intra-operative frozen sections in the diagnosis of ovarian masses. Asian Pac J Cancer Prev, 9, 737-40.

Tinelli R, Malzoni M, Cosentino F, et al (2009). Feasibility, safety, and efficacy of conservative laparoscopic treatment of borderline ovarian tumors. Fertil Steril, 92, 736-41.

Tinelli R, Tinelli A, Tinelli FG, et al (2006). Conservative surgery for borderline ovarian tumors: a review. Gynecol Oncol, 100, 185-91.

Trope CG, Kaern J, Davidson B (2012). Borderline ovarian tumours. Best Pract Res Clin Obstet Gynaecol, 26, 325-36.

Winarto H, Laihad BJ, Nuranna L (2014). Modification of cutoff values for HE4,CA125, the risk of malignancy index, and the risk of malignancy algorithm for ovarian cancer detection in Jakarta, Indonesia. Asian Pac J Cancer Prev, 15, 1949-53.

Wu TI, Lee CL, Wu MY, et al (2009). Prognostic factors predicting recurrence in borderline ovarian tumors. Gynecol Oncol, 114, 237-41.

Yinon Y, Beiner ME, Gotlieb WH, et al (2007). Clinical outcome of cystectomy compared with unilateral salpingooophorectomy as fertility-sparing treatment of borderline ovarian tumors. Fertil Steril, 88, 479-84. 